\section{P197 RETROSPECTIVE COHORT STUDY ON PAEDIATRIC APPENDICECTOMIES AND THE USE OF DIAGNOSTIC ULTRASOUND AT UNIVERSITY HOSPITAL LIMERICK}

Felicia Paluck*, Robert M O'Connell, Shona Tormey. University Hospital Limerick, Limerick, Ireland

\subsection{6/archdischild-2019-epa.552}

Background Acute abdominal pain in children remains a common presentation to A\&E departments across Ireland. Appendicectomy remains a common procedure done in children. In this study we aimed to analyse the number of paediatric appendicectomies from August 2016-August 2018 at the University Hospital Limerick (UHL), and the use of imaging in the diagnosis of acute appendicitis.

Methods Using the Hospital Inpatient Enquiry (HIPE) administrative database, all paediatric patients ( $\leq 16$ years) who underwent an appendicectomy at UHL from August 2016- August 2018 were identified retrospectively. Data were collected on each patient as follows: imaging and results, surgery (open or laproscopic appendicectomy) and histology of appendix. Negative appendicectomy rates and ultrasound diagnostic yields were calculated.

Results From August 2016- August 2018, 474 paediatric patients had an appendicectomy at UHL. Of these, 251 were male $(52.8 \%) .373$ patients had histological evidence of appendicitis (78.7\%), 55 (11.6\%) had evidence of lymphoid hyperplasia, enterobius and/or fecolith with no inflammation and $46(9.7 \%)$ had a normal appendix. There were 303 (63.9\%) laparoscopic appendicectomies and 171 (36.1\%) open appendicectomies. Only 156 (32.8\%) patients had ultrasounds. $104(66.7 \%)$ patient ultrasounds were reported inconclusive for appendicitis. $64(61.54 \%)$ of the patients with an inconclusive ultrasound yield had appendicitis.

Conclusions Ultrasound remains a poor imaging modality in diagnosing appendicitis, which may contribute to the high negative appendicectomy rates.

\section{P198 MANAGEMENT OF CHILDREN WITH DOWN SYNDROME}

Zaheera Yusuf*, Farhana Sharif. Midlands Regional Hospital Mullingar, Mullingar, Ireland

10.1136/archdischild-2019-epa.553

Down Syndrome is a chromosomal anomaly that occurs in one in every 546 live births in Ireland. ${ }^{1}$ Children with Down Syndrome require surveillance and support as they may have complex needs, including cognitive impairment, audiological issues, obstructive sleep apnoea, ophthalmic disease, cardiac defects, neurologic dysfunction, gastrointestinal disease, hip dislocation, thyroid disease, and haematological disorders. ${ }^{2}$

We reviewed the management of children with Down Syndrome attending the Mullingar, Co Westmeath Early Intervention Clinic in March 2017. Patients' charts and investigations were reviewed to determine if management was consistent with national guidelines. ${ }^{3}$ We also reviewed management in the context of the more extensive American Academy of Pediatrics Guidelines.

There were 10 patients (4 males and 6 females) attending the Early Intervention Team ranging from 3 months to 5 years of age. Eight patients were diagnosed with Down Syndrome postnatally, one was diagnosed antenatally, and one was unspecified. We identified four main areas where we were not meeting the national guidelines. First, $20 \%$ of children waited over 6 months for an echocardiogram, and $80 \%$ of children never had an electrocardiogram performed. Second, dental advice or infective endocarditis advice was not documented at any visit. Third, there was a delay in referral for ophthalmology assessment for two patients at their $18-24$ months visit. Fourth, referrals to audiology services were variable and delayed up to 3 years of age. Lastly, the AAP advise a more comprehensive assessment of children at their routine appointments. ${ }^{4}$ While half of patients had a coeliac screen organised, patients rarely had an FBC, CRP, or ferritin performed. Vaccinations, including RSV prophylaxis and influenza vaccine, were discussed with two patients. Families were not given cspine advice, or asked about neurological dysfunction or obstructive sleep apnoea.

Our local practice would be improved with dentition review, and early referrals to and reviews by cardiology, ophthalmology, and audiology. We would suggest the introduction of a clinical template to guide appointments, and a review of the AAP guidelines, with a view to consider including their recommendations into Irish practice.

\section{REFERENCES}

1. Irish Health. http://www.irishhealth.com/article.html?con=64. 2006.

2. Bull MJ, and the Committee on Genetics. Clinical Report-Health Supervision of Children With Down Syndrome. American Academy of Pediatrics. Pediatrics Volume 128 (2): 393-406, 2011.

3. Down Syndrome Ireland. Suggested Schedule of Health Checks. https://downsyndrome.ie/wp-content/uploads/2017/01/Medical-Management-Guidelines-SuggestedSchedule-of-Health-Checks.pdf. 2017.

4. Voigt RG, Macias MM, and Myers SM. AAP Developmental and Behavioural Paediatrics. American Academy of Pediatrics, 2011.

\section{P199 HUDDLING FOR SAFETY: THE FIRST IRISH PAEDIATRIC SAFE COLLABORATIVE}

${ }^{1}$ Rachel MacDonell ${ }^{*},{ }^{2}$ John Fitzsimons, ${ }^{3}$ Peter Lachman, ${ }^{1}$ Alf Nicholson. ${ }^{1} R C P I$, Dublin, Ireland; ${ }^{2}$ HSE, Dublin, Ireland; ${ }^{3}$ ISQUA, Dublin, Ireland

10.1136/archdischild-2019-epa.554

Situation Awareness for Everyone (SAFE) is a collaborative programme developed by the Royal College of Paediatrics and Child Health UK to support clinical teams to improve communication, build a safety based culture and deliver better outcomes for children and young people.

The programme includes Quality Improvement (QI) theory, situation awareness methodology and planning of safety huddles on the wards. It has been implemented in over 50 teams across the UK and the Quality Improvement Department of the Royal College of Physicians of Ireland are delighted to bring this initiative to Ireland.

A National Paediatric Early Warning System (PEWS) was endorsed by the Irish Minister for Health in December 2015. The supporting National Clinical Guideline recommends that hospitals 'support additional safety practices that enhance the PEWS and lead to greater situation awareness among clinicians and multidisciplinary teams, such as incorporating briefings, safety pause and huddles into practice'. Programmes such as SAFE that use quality improvement methods and patient safety science were specified as appropriate to assist hospitals to collaborate in addressing these challenges.

11 consultant-led teams from across Ireland are participating in this SAFE Collaborative. Teams consist mostly of 
frontline paediatric clinical care providers (Consultants in Paediatric Medicine, Paediatric Nurses, Pharmacists, Dieticians, Hospital Senior Management and Non-Consultant Hospital Doctors). At a series of face-to-face sessions, teams receive mentoring in QI methodology by RCPI QI Faculty, through the IHI Breakthrough Series Collaborative Model, to develop local SAFE improvement projects impacting on

- Reducing avoidable error and harm to acutely unwell children

- Improving communication between all individuals involved in a child's care

- Improving working culture for healthcare staff providing care to children

- Increasing involvement of parents, children and young people in their care.

The teaching faculty includes active patient representation through parent involvement. Participating teams are encouraged to engage with children, parents and carers to guide their improvement efforts.

Teams collect a concise monthly dataset to facilitate aggregate and comparative measures on paediatric clinical outcomes. Teams are encouraged to use this data, and to collect other necessary data to inform the outcome, process and balancing measures pertinent to the areas upon which they are focusing their improvement efforts.

The National SAFE Improvement Collaborative is in its early stages. However, at this point, experience with similar national collaborative projects indicates that QI methodology will be used effectively to generate improvements to positively impact paediatric patient safety outcomes, through site specific changes.

\section{P200 MANAGEMENT OF HENOCH SCHONLEIN PURPURA (HSP)}

${ }^{1}$ Lubna Mahmood*, ${ }^{2}$ Farah Zulfiqar, ${ }^{3}$ Saira Khalid, ${ }^{4}$ Michelle Dillon. 'St.Luke's Hospital, Kilkenny, Ireland; '2St.Luke's Hospital, Kilkenny, Ireland; ${ }^{3}$ St. Luke's Hospital, Kilkenny, Ireland; 'St. Luke's Hospital , Kilkenny, Ireland

10.1136/archdischild-2019-epa.555

Introduction HSP is a common vasculitis of childhood. It is immune mediated. Aetiology is unknown but the history often identifies a preceding throat/URTI infection.

Agreed criteria for HSP diagnosis are:

Palpable purpura (mandatory) in the presence of atleast one of the following;

Diffuse abdominal pain

Acute arthritis or arthralgia

Renal involvement (haematuria +/_ proteinuria)

Renal biopsy showing predominantly IgA deposition.

Diagnosis is usually on clinical grounds . Bloods are needed to rule out other diagnoses. All patients need BP, urine dipstick, weight and height measurements.

General supportive measures and simple analgesia is all that is required . HSP is usually self limiting. 33\% will have relapses/recurrence of symptoms. HSP accounts for $3 \%$ of all patients with end-stage renal failure .

Aim To look at management of HSP in our unit during the last six months, develop local guidelines and then re audit to monitor compliance with guidelines.

Method We carried out a retrospective observational charts review of all children presenting with HSP.
Results No standard guidelines were being followed. Followup practices differed between teams. We developed local guidelines for the management of HSP.

If the initial urinalysis is normal or only reveals microscopic haematuria, follow up involves : clinical review, BP measurement and early morning urinalysis at these recommended time intervals :

1. Weekly for the first month

2. Fortnightly from weeks 5 to 12

3. Single review at 6 and 12 months

4. Return to (1) if there is disease flare up

Referral to Paediatric Nephrologist is warranted if there is : Macroscopic haematuria more than 5 days.

Persistent microscopic haematuria beyond 12 months.

Persistent proteinuria.

Hypertension

Abnormal renal function

Nephrotic syndrome

Nephritic syndrome

We carried out retrospective charts review again in the following six months. There were 7 cases of HSP noted . 5 patients had HSP with no renal involvement and were followed according to local protocol. It was noted that three of these patients had unnecessary blood tests done including Coagulation profile. One patient was not followed up after after the initial presentation due to miscommunication but later reviewed at 3 months.

One patient has mild HSP Nephritis with persistent proteinurea and macroscopic haematuria and was referred to the nephrologist. Her symptoms resolved completely.

We will continue to audit our practise. In addition to monitoring renal status we also aim to avoid unnecessary blood tests. Streaming our followup may identify early markers of renal disease in this group of children.

\section{P201 AN AUDIT OF THE COMPLIANCE WITH A NEWLY INTRODUCED 'ELECTRONIC PATIENT DISCHARGE' SYSTEM IN THE DEPARTMENT OF PAEDIATRICS, UNIVERSITY HOSPITAL LIMERICK (UHL)}

Uzair Athar Khan*, Katie Flyn, Anne Murie Murphy, Siobhan Gallagher. UHL, Dooradoyle Limerick, Ireland

\subsection{6/archdischild-2019-epa.556}

Background Electronic discharge application is a multi-tenant based application (any hospital in the country can use it if desired). It first started in June 2017. The primary reason for introducing it was to aid communication between the hospital and General Practitioners (GP).The focus of this project was to provide a streamlined method for Non- Consultant Hospital Doctors (NCHDs) to easily generate a patient discharge summary letter in an electronic format that can be reviewed and signed off by their Consultants. This discharge summary is then available for sending to a patient's GP via electronic means or in printed hardcopy.

These requirements are based in part on the HIQA 'National Standard for Patient Discharge Summary information document. This helps in terms of ensuring that all relevant data is captured and fed back to the patients GP in a clear, concise and timely manner .In addition to supporting the primary goal, this will further support a myriad of 